\title{
Reactivity of Hydrogen-Helium and Hydrogen-Nitrogen Mixtures at High Pressures
}

\author{
Robin Turnbull, ${ }^{1}$ Mary-Ellen Donnelly, ${ }^{2}$ Mengnan Wang, ${ }^{2}$ Miriam Peña-Alvarez, ${ }^{1}$ \\ Cheng Ji, ${ }^{2,3}$ Philip Dalladay-Simpson, ${ }^{2}$ Ho-kwang Mao, ${ }^{2}$ Eugene Gregoryanz, ${ }^{1,2}$ and Ross T. Howie ${ }^{2 *}$ \\ ${ }^{1}$ School of Physics and Astronomy and Centre for Science at Extreme Conditions, \\ University of Edinburgh, Edinburgh EH9 3FD, United Kingdom \\ ${ }^{2}$ Center for High Pressure Science and Technology Advanced Research, Shanghai 201203, People's Republic of China \\ ${ }^{3}$ High Pressure Collaborative Access Team, Geophysical Laboratory, Carnegie Institution of Washington, \\ Argonne, Illinois 60439, USA
}

(Received 9 July 2018; revised manuscript received 17 August 2018; published 9 November 2018)

\begin{abstract}
Through a series of Raman spectroscopy studies, we investigate the behavior of hydrogen-helium and hydrogen-nitrogen mixtures at high pressure across a wide range of concentrations. We find that there is no evidence of chemical association or increased miscibility of hydrogen and helium in the solid state up to pressures of $250 \mathrm{GPa}$ at $300 \mathrm{~K}$. In contrast, we observe the formation of concentration-dependent $\mathrm{N}_{2}-\mathrm{H}_{2}$ van der Waals solids, which react to form $\mathrm{N}-\mathrm{H}$ bonded compounds above $50 \mathrm{GPa}$. Through this combined study, we can demonstrate that the recently reported chemical association of $\mathrm{H}_{2}-\mathrm{He}$ can be attributed to significant $\mathrm{N}_{2}$ contamination and subsequent formation of $\mathrm{N}_{2}-\mathrm{H}_{2}$ compounds.
\end{abstract}

DOI: 10.1103/PhysRevLett.121.195702

Understanding the behavior of molecular mixtures under pressure is of great importance in many scientific fields, varying from chemistry to the studies of internal structures of astronomical bodies [1,2]. A wide range of phenomena have been observed in high-pressure molecular mixtures, such as phase separation, cocrystallization, host-guest structures, and chemical reaction [3-6]. Since the discovery of solid van der Waals compounds in the high-pressure heliumnitrogen system, binary mixtures of elemental gases have attracted much attention both experimentally and theoretically [7-11]. Of particular importance are binary mixtures of the four lightest elemental gasses: $\mathrm{H}_{2}, \mathrm{He}, \mathrm{N}_{2}$, and $\mathrm{O}_{2}$, which have been widely explored at high pressure [12-19]. Recently, there has been renewed interest in studies of both the hydrogen-helium and hydrogen-nitrogen systems at high pressure, with a focus on investigating the possible synthesis of compounds through the reaction of the constituent molecules [20-25].

$\mathrm{H}_{2}$ and $\mathrm{He}$ are predicted to be chemically inert towards each other across wide pressure-temperature $(P-T)$ and concentration regimes [26-32]. Theoretical simulations, motivated by potential miscibility within the Jovian planets, find evidence that, even at these extreme conditions, hydrogen and helium remain phase separated or at most exhibit partial miscibility. Because of the theoretical predictions of no chemical reactivity between hydrogen and helium, there have been few experimental studies on mixtures. Early studies exploring the eutectic phase diagram of hydrogen-helium mixtures found that the hydrogen intramolecular vibrational mode in the two-fluid state exhibited a marked frequency increase in He-rich concentrations, which was explained semiquantitatively by a helium compressional effect [13]. However, in the solid state, the two species were shown to be completely immiscible up to $15 \mathrm{GPa}$. This observation of immiscibility was utilized to grow single crystals of $\mathrm{H}_{2}$ and measure the equation of state up to $100 \mathrm{GPa}$ without an observable chemical reaction between the two [33]. A recent highpressure study exploring $\mathrm{H}_{2}-\mathrm{He}$ interactions as a function of mixture concentration, reported the unprecedented appearance of hydrogen-helium solids at pressures below $75 \mathrm{GPa}$ [20]. Through the appearance of a vibrational Raman band at a frequency similar to that calculated for the $\mathrm{H}-\mathrm{He}$ stretch in a linear H-He-F molecule [34], the authors claim the formation of $\mathrm{H}-\mathrm{He}$ bonds [20]. These results are surprising given that the corresponding $P-T$ regime has already been explored both experimentally and theoretically $[13,16]$.

In contrast to $\mathrm{H}_{2}$ - $\mathrm{He}$ mixtures, the $\mathrm{H}_{2}-\mathrm{N}_{2}$ system exhibits particularly rich physics under compression, which is strongly dependant on both pressure and $\mathrm{N}_{2}: \mathrm{H}_{2}$ composition. Two van der Waals compounds have been reported to form at pressures above $\sim 7 \mathrm{GPa}:\left(\mathrm{N}_{2}\right)_{6}\left(\mathrm{H}_{2}\right)_{7}$ and $\mathrm{N}_{2}\left(\mathrm{H}_{2}\right)_{2}$ [22,25]. At pressures between 35 and $50 \mathrm{GPa}$, these van der Waals compounds react to form $\mathrm{N}-\mathrm{H}$ bonded solids, which cannot be characterized solely by known hydronitrogen compounds. On decompression, these solids transform to hydrazine $\left(\mathrm{N}_{2} \mathrm{H}_{4}\right)$ below $10 \mathrm{GPa}$ [22-25].

In this study, we have comprehensively investigated the pressure induced reactivity of $\mathrm{H}_{2}-\mathrm{He}$ and $\mathrm{H}_{2}-\mathrm{N}_{2}$ mixtures as a function of mixture composition through Raman spectroscopy. Hydrogen and helium remain nearly immiscible across all concentrations up to pressures of $250 \mathrm{GPa}$, with no formation of van der Waals compounds nor any 
chemical reactivity across all mixture concentrations studied. Even at the extreme densities of hydrogen phase IV, which is thought to adopt a complex layered structure, no chemical association is observed [35]. In contrast, modest pressures readily induce the formation of $\mathrm{H}_{2}-\mathrm{N}_{2}$ van der Waals compounds, which on compression above $50 \mathrm{GPa}$, react to form $\mathrm{N}-\mathrm{H}$ bonded compounds that remain stable on subsequent decompression. Through this combined study of both systems, we demonstrate that the recently reported chemical association between $\mathrm{H}_{2}$ and $\mathrm{He}$ can be described by the formation of $\mathrm{N}_{2}-\mathrm{H}_{2}$ compounds due to significant $\mathrm{N}_{2}$ contamination of the $\mathrm{H}_{2}-\mathrm{He}$ mixtures used in that study [20].

Research grade $(99.9995 \%)$ hydrogen-helium mixtures with molar hydrogen concentrations of $10 \%, 20 \%, 30 \%$, and $50 \%$ were obtained commercially. Hydrogen-nitrogen compositions were prepared in-house from research grade $(99.9995 \%) \mathrm{H}_{2}$ and $\mathrm{N}_{2}$, with molar hydrogen concentrations of $28 \%, 50 \%, 59 \%$, and $75 \%$ as determined from the relative partial pressures. The mixtures were given several days to homogenize before being gas loaded into diamond anvil cells (DACs). All samples were gas loaded into the DACs at a pressure of $200 \mathrm{MPa}$. Raman spectroscopy was conducted using a custom-built microfocused system, using the 514 and $647 \mathrm{~nm}$ excitation wavelengths (see Supplemental Material for further details [36]). Powder x-ray diffraction measurements of $\mathrm{H}_{2}-\mathrm{N}_{2}$ mixtures were collected at beam line P02.2, PETRA III, Germany using a monochromatic beam of $\lambda=0.484693 \AA$ and recorded on a Mar 345 image plate area detector. Pressure was determined using the ruby fluorescence scale below $100 \mathrm{GPa}$ and the stressed Raman edge of diamond at higher pressures [41,42].

Upon loading the samples of hydrogen-helium, all concentrations exhibited only the Raman modes that can be attributed to rotational modes (rotons) and vibrational modes (vibrons) of $\mathrm{H}_{2}$ (see Fig. 1 and Supplemental Material Figs. S1-S7 [36]). In the fluid state (below $6 \mathrm{GPa}$ ), the two species are mixed well and the intensity and frequency of the hydrogen mode is constant when measured at different points across the sample chamber. The hydrogen vibron in the fluid sample does, however, exhibit an increase in Raman frequency, when compared with the pure species, which increases with greater helium concentrations (see inset of Fig. 1). This is in good agreement with previous studies on the binary phase diagram [13]. In addition, we observe significant broadening of the $\mathrm{H}_{2}$ vibron with pressure in the fluid-fluid phase compared with the pure species (see Fig. S8 [36]). At $4 \mathrm{GPa}$, there is a fivefold increase in the full width at half maximum (FWHM) of the $\mathrm{H}_{2}$ vibron compared to pure $\mathrm{H}_{2}$ (a comparable vibron width to phase III of $\mathrm{H}_{2}$ at $200 \mathrm{GPa}$ and $300 \mathrm{~K}$ ), and the rate of broadening is independent of concentration.

At pressures greater than $5.2 \mathrm{GPa}$, the immiscibility of $\mathrm{H}_{2}$ and $\mathrm{He}$ becomes evident by the visible phase separation

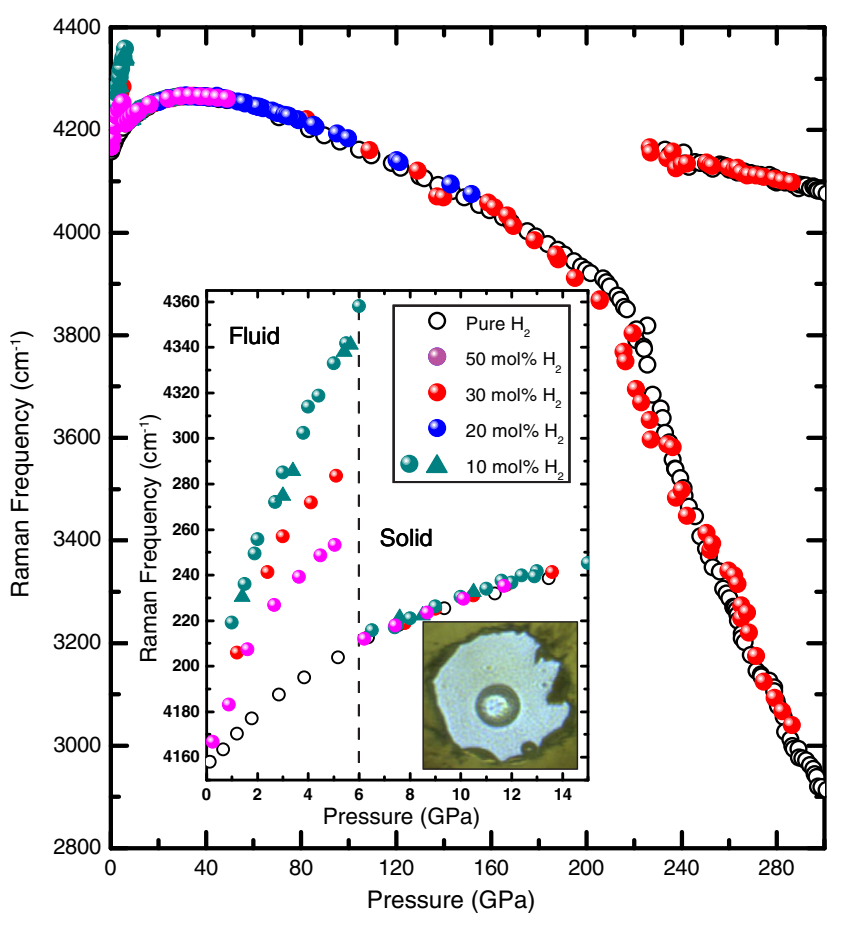

FIG. 1. Raman frequency vs pressure for different $\mathrm{H}_{2}: \mathrm{He}$ concentrations up to $285 \mathrm{GPa}$. Circles and triangles represent data collected on compression and decompression, respectively. (Inset) Raman frequency vs pressure for different $\mathrm{H}_{2}$ : He concentrations up to $20 \mathrm{GPa}$. Photomicrograph of a $10 \mathrm{~mol} \% \mathrm{H}_{2}$ in He sample at $15 \mathrm{GPa}$. The single crystal of $\mathrm{H}_{2}$, located in the center of the sample chamber, is clearly phase separated from the surrounding He medium.

as hydrogen enters the solid state. Across all concentrations, the Raman frequency of the hydrogen vibron reverts to the same frequency as that exhibited by the pure species under the same $P-T$ conditions, and the frequency shift is completely reversible on decompression back into the fluid state. Slow sample compression between the $\mathrm{H}_{2}$ and $\mathrm{He}$ solidifications pressures causes nucleation of hydrogen crystals, at the edge of the sample chamber, which coalesce over time (see insert of Fig. 1 and Supplemental Material Fig. S9 [36]). On rapid compression from the fluid-fluid to the solid-solid state, small $\mathrm{H}_{2}$ crystallites form across the whole sample chamber. In the majority of our rapid compression experimental runs, we still observed a weak $\mathrm{H}_{2}$ vibron in the $\mathrm{He}$ medium, which we attribute to $\mathrm{H}_{2}$ molecules trapped in the He lattice. There is no detectable $\mathrm{H}_{2}$ vibron in the $\mathrm{He}$ medium of low- $\mathrm{H}_{2}$ concentration samples, which are held below the $\mathrm{He}$ solidification pressure for a period of weeks. Across all concentrations there is negligible frequency difference between the $\mathrm{H}_{2}$ vibron in the He medium compared with the bulk $\mathrm{H}_{2}$. To rule out any kinetic effects, samples at each concentration were held in the $\mathrm{H}_{2}$-He fluid-fluid, solid-fluid and solidsolid states for a period of one week and no changes were observed with time. One sample at a concentration $20 \mathrm{~mol}$ 
$\% \mathrm{H}_{2}$ was held for a period of eight years at a pressure of $120 \mathrm{GPa}$ with no evidence of a chemical reaction.

At $300 \mathrm{~K}$, pure hydrogen has been shown to go through a phase transition sequence of I-III above $180 \mathrm{GPa}$ and IIIIV above $225 \mathrm{GPa}$ [35]. Phase IV is believed to adopt a twolayer molecular structure, giving rise to two distinct vibrational modes. One would expect that hydrogen in this phase would be more reactive, due to the much shorter molecular lifetime. It is also known that, above $200 \mathrm{GPa}, \mathrm{H}_{2}$ and $\mathrm{D}_{2}$ tend to form a molecular alloy with each other, which does not happen at lower pressures [43]. Figure 1 (and Fig. S10 [36]) shows the hydrogen vibron frequency as a function of pressure for a $30 \%$ hydrogen in helium mixture up to the conditions of phase IV. We see only slight deviation in the vibron frequency when compared to pure $\mathrm{H}_{2}$ and the deviation is well within experimental error of pressure determination.

Our data clearly show that, over a broad pressure regime and over wide-ranging concentrations, there is no evidence of any chemical interactions between $\mathrm{H}_{2}$ and $\mathrm{He}$, and they remain nearly immiscible up to pressures of $250 \mathrm{GPa}$. This is in disagreement with the recent results reported in Ref. [20], which reported chemical association between $\mathrm{H}_{2}$ and He. The evidence for chemical association was primarily through the appearance of a Raman band at $\sim 2330 \mathrm{~cm}^{-1}$ upon loading of the sample, which the authors attribute to a $\mathrm{H}-\mathrm{He}$ bonded molecule. We do not observe this mode across all concentrations studied (see Fig. 1 and Supplemental Material Figs. S1-S7 [36]). However, the $\mathrm{N}_{2}$ molecule exhibits the same characteristic vibrational frequency $\left(2330 \mathrm{~cm}^{-1}\right.$ at $\left.0.5 \mathrm{GPa}\right)$ as that of the claimed $\mathrm{H}-\mathrm{He}$ vibrational mode. Although the authors of Ref. [20] make efforts to rule out $\mathrm{N}_{2}$ contamination by comparing their data to $\mathrm{N}_{2}$ (and $\mathrm{N}_{2}$ in $\mathrm{He}$ ) Raman vibrational frequencies in the solid state, they crucially do not consider the possibility of $\mathrm{N}_{2}-\mathrm{H}_{2}$ interactions. As such, we present our own data, investigating the chemical interactions in $\mathrm{H}_{2}$ and $\mathrm{N}_{2}$ mixtures, in the pressure regime at which $\mathrm{H}_{2}-\mathrm{He}$ chemical association was reported.

In the fluid state, hydrogen-nitrogen mixtures are characterized only by the vibrational modes exhibited in the pure $\mathrm{H}_{2}$ and $\mathrm{N}_{2}$ species (see Fig. S11 [36]). Across all concentrations studied, the $\mathrm{H}_{2}$ vibron exhibits a redshift in frequency, which increases with greater $\mathrm{N}_{2}$ concentration. In contrast, the $\mathrm{N}_{2}$ vibron shows little effect by concentration in the fluid, and the pressure dependence follows closely with the pure species [see Fig. 2(b)]. The solidification pressure of pure $\mathrm{N}_{2}$ is $2 \mathrm{GPa}$, while it is $5.5 \mathrm{GPa}$ for hydrogen. Interestingly, in mixtures of $\mathrm{N}_{2}$ and $\mathrm{H}_{2}$, all concentrations are homogeneous fluids below $8 \mathrm{GPa}$, before solidifying into $\mathrm{N}_{2}-\mathrm{H}_{2}$ van der Waals compounds that exhibit no $\mathrm{N}-\mathrm{H}$ bond vibrational modes.

Figure 2(a) shows the Raman spectra for each $\mathrm{N}_{2}: \mathrm{H}_{2}$ concentration at $\sim 40 \mathrm{GPa}$ compared with the pure species. At $75 \mathrm{~mol} \% \mathrm{H}_{2}$, we can identify the formation of both
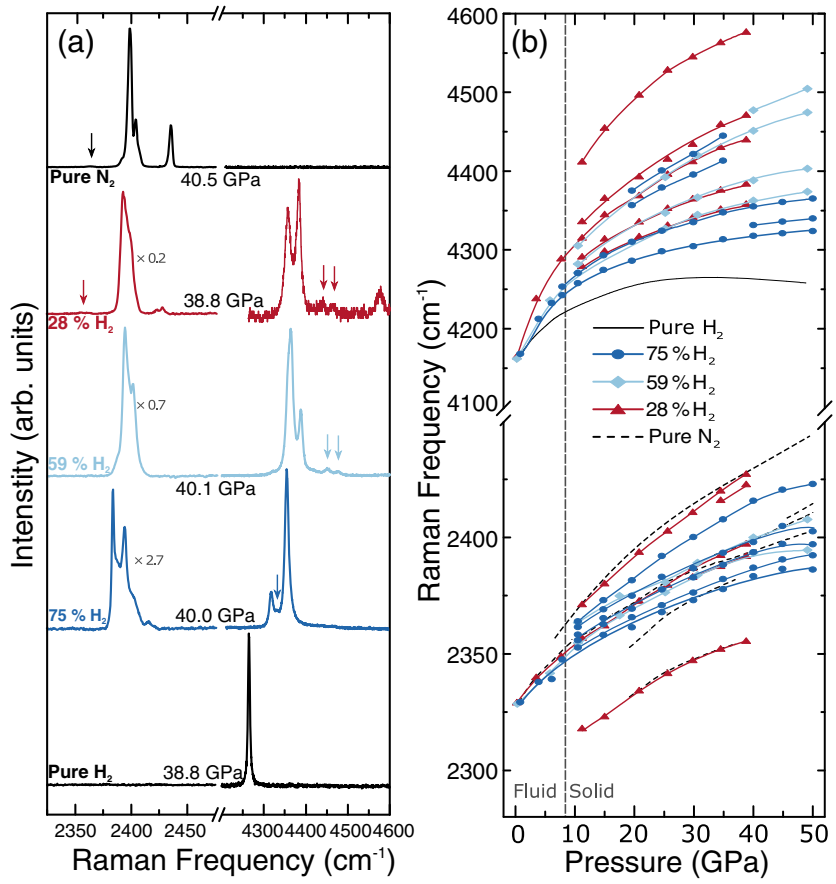

FIG. 2. (a) Evolution of the Raman spectra with composition in the solid state at around $40 \mathrm{GPa}$ for the nitrogen-hydrogen compositions of 28, 59, and $75 \mathrm{~mol} \% \mathrm{H}_{2}$. The pure species are included for comparison in black. (b) Raman frequency vs pressure plots corresponding to the data shown in (a). Solid and dashed black lines correspond to pure $\mathrm{H}_{2}$ and pure $\mathrm{N}_{2}$, respectively. Arrows indicate weak Raman bands.

$\left(\mathrm{N}_{2}\right)_{6}\left(\mathrm{H}_{2}\right)_{7}$ and $\mathrm{N}_{2}\left(\mathrm{H}_{2}\right)_{2}$ through our powder x-ray diffraction measurements (Fig. S12 [36]), which are consistent with structures previously identified through single crystal $\mathrm{x}$-ray diffraction analysis $[22,25]$. The coexistence of these compounds is different with respect to the previously reported binary phase diagram, which reported an overlap region between 54 and $66 \mathrm{~mol} \%$ [22]. At lower $\mathrm{H}_{2}$ concentrations of $59 \%$ and $28 \%$, we see only $\left(\mathrm{N}_{2}\right)_{6}\left(\mathrm{H}_{2}\right)_{7}$ and $\delta$ - $\mathrm{N}_{2}$ in the diffraction patterns.

Figure 3 compares the Raman frequencies of our 75 and $59 \mathrm{~mol} \% \mathrm{H}_{2}-\mathrm{N}_{2}$ mixtures with that of 50 and $10 \mathrm{~mol} \%$ $\mathrm{H}_{2}-\mathrm{He}$ mixtures in Ref. [20]. Below $7 \mathrm{GPa}$, there is a close match, in both the frequency and pressure dependency, between the claimed $\mathrm{H}-\mathrm{He}$ vibrational mode and that of $\mathrm{N}_{2}$ in the $\mathrm{H}_{2}-\mathrm{N}_{2}$ mixture. It is unlikely that a $\mathrm{H}-\mathrm{He}$ vibrational mode would have the same frequency dependence over a $7 \mathrm{GPa}$ interval as a triple-bonded nitrogen molecule. At higher pressures, we find that the vibrational Raman modes of the $S_{2}$ phase of Ref. [20] in a 5:5 $\mathrm{H}_{2}$-He mixture closely match that of a $3: 2 \mathrm{H}_{2}-\mathrm{N}_{2}$ mixture across the whole pressure regime studied. At the mixture ratio of $3: 2$, $\mathrm{N}_{2}\left(\mathrm{H}_{2}\right)_{2}$ is the dominant compound but it coexists with $\left(\mathrm{N}_{2}\right)_{6}\left(\mathrm{H}_{2}\right)_{7}$. At $12 \mathrm{GPa}$, the Raman mode at $\sim 4265 \mathrm{~cm}^{-1}$ corresponds to the $\mathrm{H}_{2}$ vibron in $\mathrm{N}_{2}\left(\mathrm{H}_{2}\right)_{2}$, while the higher frequency vibron at $\sim 4288 \mathrm{~cm}^{-1}$ corresponds to the most intense $\mathrm{H}_{2}$ vibrons in $\left(\mathrm{N}_{2}\right)_{6}\left(\mathrm{H}_{2}\right)_{7}$. In the high-He content 


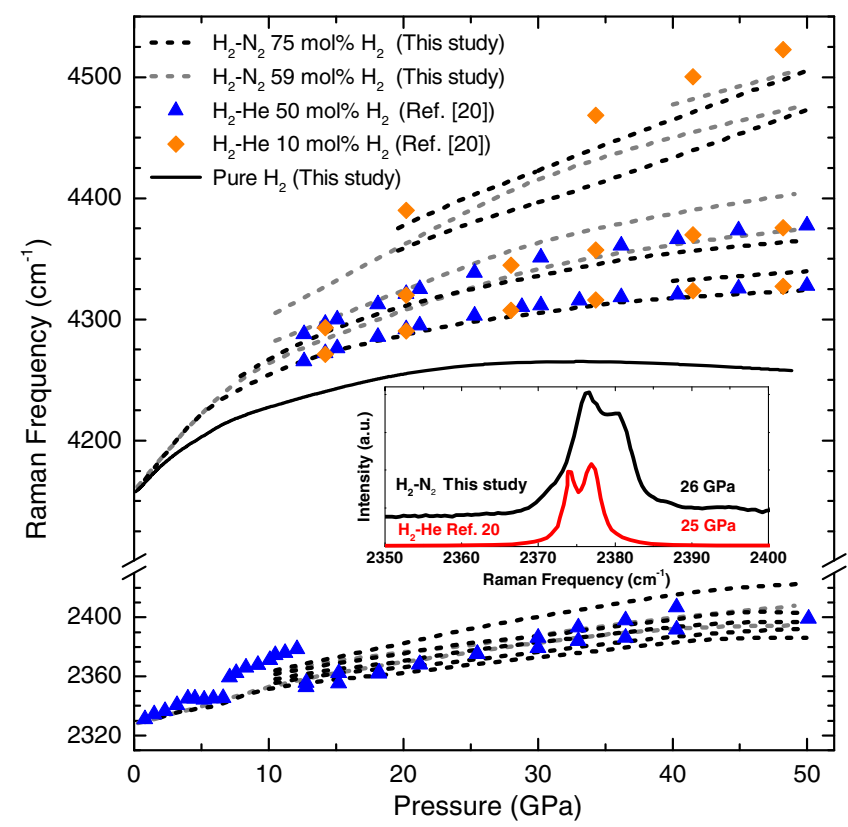

FIG. 3. A comparison of the Raman frequencies as a function of pressure between our data on $\mathrm{H}_{2}-\mathrm{N}_{2}$ mixtures (dashed lines), pure $\mathrm{H}_{2}$ (black line), and the $\mathrm{H}_{2}-\mathrm{He}$ compound (known as " $\mathrm{S}_{2}$ ") reported in Ref. [20] (symbols). (Inset) Comparison between "H-He" vibrons from Ref. [20] and the $\mathrm{N}_{2}$ vibrons from a 3:2 $\mathrm{H}_{2}-\mathrm{N}_{2}$ mixture.

mixtures, there is a third $\mathrm{H}_{2}$ vibrational mode at frequencies $\sim 4390 \mathrm{~cm}^{-1}$, which corresponds to the second most intense Raman band of $\left(\mathrm{N}_{2}\right)_{6}\left(\mathrm{H}_{2}\right)_{7}$. The behavior of the $\mathrm{N}_{2}$ vibrons is also very different in $\mathrm{H}_{2}-\mathrm{N}_{2}$ compounds than in either pure $\mathrm{N}_{2}$ or $\mathrm{N}_{2}: \mathrm{He}$ compounds. We find excellent agreement, shown in Fig. 3 (and Fig. S13 [36]), between the $\mathrm{N}_{2}$ stretches in $\mathrm{N}_{2}\left(\mathrm{H}_{2}\right)_{2}$ and the claimed $\mathrm{H}-\mathrm{He}$ vibrational mode of Ref. [20].

Above the critical pressure of $50 \mathrm{GPa}$ at room temperature, all samples exhibited loss of intensity of the hydrogen and nitrogen Raman vibrational bands over hour-long timescales (see Fig. 4). The loss of vibron intensity occurred simultaneously with the emergence of a broad asymmetric peak centered around $3400 \mathrm{~cm}^{-1}$ (highlighted in blue in Fig. 4). The broad asymmetric peak around $3400 \mathrm{~cm}^{-1}$ can be attributed to the vibrational modes of $\mathrm{N}-\mathrm{H}$ bonded compounds; however the overall appearance does not match the known hydronitrogen compounds [40,44]. This difference could be attributed to the formation of a combination of azanes and/or the presence of excess reactants. This solid phase remained on decompression until $10 \mathrm{GPa}$, after which the broad peak around $3400 \mathrm{~cm}^{-1}$ evolved into two sharp peaks accompanied by four lower frequency modes (see Fig. 4) unambiguously identifying hydrazine $[44,45]$. Solid hydrazine was observed on decompression below $10 \mathrm{GPa}$ in all isothermal compression-decompression experiments and across all concentrations studied.

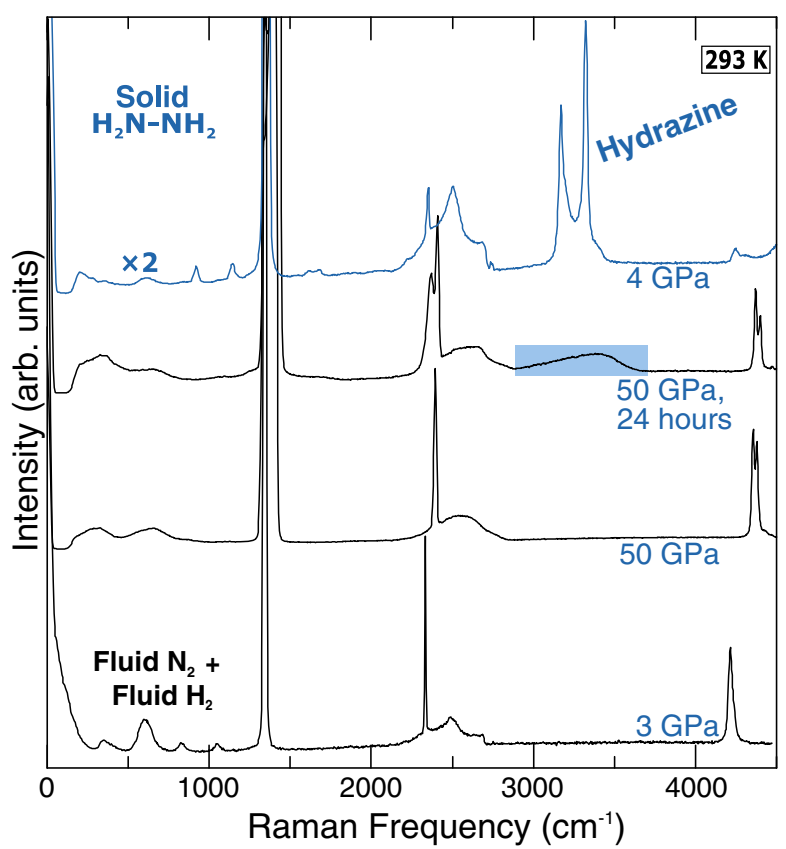

FIG. 4. Raman spectra on compression of a $50 \mathrm{~mol} \% \mathrm{H}_{2}$ composition demonstrating the time-dependent chemical reaction of $\mathrm{N}_{2}$ and $\mathrm{H}_{2}$ above $50 \mathrm{GPa}$. The blue shaded region indicates the formation of $\mathrm{N}-\mathrm{H}$ bonds. The blue spectrum shows the recovery of hydrazine on decompression to $4 \mathrm{GPa}$. For a comparison between these data and Ref. [20], see Figs. S14 and S15 [36].

The spectral changes shown in Fig. 4 were also observed in Ref. [20], but were interpreted as the formation of another H-He solid (" $S_{1}$ "). We find good agreement between the spectra of this solid and the N-H compounds we observe to form (see Figs. S14 and S15). This, together with the above analysis, shows that the previously reported chemical association between $\mathrm{H}_{2}$ and He could be attributed to significant nitrogen contamination of the samples. The authors of Ref. [20] produce the gas mixtures themselves, and it is possible that the $\mathrm{N}_{2}$ contaminant is introduced at the initial gas mixing stage or during sample loading. Our own experience in producing gas mixtures demonstrates that great care needs to be taken to ensure that the ballast volume between gas bottles in the mixture setup is adequately purged with the constituent gases $[4,43]$. We have sometimes observed trace nitrogen contamination from air due to this, and in these cases, we would disregard the contaminated gas bottle. In this study, we obtain our $\mathrm{H}_{2}-\mathrm{He}$ mixtures commercially with guaranteed levels of purity and do not reproduce any of the observations of Ref. [20].

Our results show that, even at extreme compressions, $\mathrm{H}_{2}$ and $\mathrm{He}$ remain immiscible and chemically inert towards each other, both properties that will prove advantageous for future structural studies of phase IV hydrogen. In agreement with previous theoretical results, it is likely that extreme $P-T$ conditions in excess of that in the interiors of 
Jovian planets would be required for $\mathrm{H}_{2}$ and $\mathrm{He}$ to become miscible, let alone form chemical bonds.

Parts of this research were carried out at P02.2 at DESY, a member of the Helmholtz Association (HGF). We would like to thank H.-P. Liermann and K. Glazyrin for their assistance. R. T. acknowledges studentship funding from EPSRC Grants No. EP/L504956/1 and No. EP/M506515/1. M.P.-A. would like to acknowledge the support of the European Research Council (ERC) Grant Hecate Reference No. 695527. We would also like to thank M. Frost and V. Afonina for help in experiments and J. Binns for useful discussions.

*ross.howie@hpstar.ac.cn

[1] A. Dewaele N. Worth, C. J. Pickard, R. J. Needs, S. Pascarelli, O. Mathon, M. Mezouar, and T. Irifun, Nat. Chem. 8, 784 (2016).

[2] J. S. Loveday R. J. Nelmes, M. Guthrie, S. A. Belmonte, D. R. Allan, D. D. Klug, J. S. Tse, and Y. P. Handa, Nature (London) 410, 661 (2001).

[3] M. A. Morales, S. Hamel, K. Caspersen, and E. Schwegler, Phys. Rev. B 87, 174105 (2013).

[4] R. T. Howie, R. Turnbull, J. Binns, M. Frost, P. DalladaySimpson, and E. Gregoryanz, Sci. Rep. 6, 34896 (2016).

[5] O. Degtyareva, V. V. Struzhkin, and R. J. Hemley, Solid State Commun. 141, 164 (2007).

[6] B. Guigue, A. Marizy, and P. Loubeyre, Phys. Rev. B 95, 020104 (2017).

[7] W. L. Vos, L. W. Finger, R. J. Hemley, J. Z. Hu, H. K. Mao, and J. A. Schouten, Nature (London) 358, 46 (1992).

[8] C. Cazorla, D. Errandonea, and E. Sola, Phys. Rev. B 80, 064105 (2009).

[9] C. Cazorla and D. Errandonea, Phys. Rev. B 81, 104108 (2010).

[10] M. Somayazulu, P. Dera, A. F. Goncharov, S. A. Gramsch, P. Liermann, W. Yang, Z. Liu, H.-k. Mao, and R. J. Hemley, Nat. Chem. 2, 50 (2010).

[11] C. Ji et al., Proc. Natl. Acad. Sci. U.S.A. 114, 3596 (2017).

[12] P. Loubeyre, R. Le Toullec, and J.-P. Pinceaux, Phys. Rev. B 32, 7611 (1985).

[13] P. Loubeyre, R. Le Toullec, and J. P. Pinceaux, Phys. Rev. B 36, 3723 (1987).

[14] P. Loubeyre, R. Le Toullec, and J. P. Pinceaux, J. Phys. Condens. Matter 3, 3183 (1991).

[15] P. Loubeyre, R. LeToullec, and J. P. Pinceaux, Phys. Rev. B 45, 12844 (1992).

[16] P. Loubeyre, and R. LeToullec, Nature (London) 378, 44 (1995).

[17] H. Olijnyk and A. P. Jephcoat, J. Phys. Condens. Matter 9, 11219 (1997).

[18] D. Sihachakr and P. Loubeyre, Phys. Rev. B 70, 134105 (2004).
[19] G. Weck, A. Dewaele, and P. Loubeyre, Phys. Rev. B 82, 014112 (2010)

[20] J. Lim and C.-S. Yoo, Phys. Rev. Lett. 120, 165301 (2018).

[21] M. Kim and C.-S. Yoo, J. Chem. Phys. 134, 044519 (2011).

[22] D. K. Spaulding, G. Weck, P. Loubeyre, F. Datchi, P. Dumas, and M. Hanfland, Nat. Commun. 5, 5739 (2014).

[23] H. Wang, M. I. Eremets, I. Troyan, H. Liu, Y. Ma, and L. Vereecken, Sci. Rep. 5, 13239 (2015).

[24] A. F. Goncharov et al., J. Chem. Phys. 142, 214308 (2015).

[25] D. Laniel, V. Svitlyk, G. Weck, and P. Loubeyre, Phys. Chem. Chem. Phys. 20, 4050 (2018).

[26] J. E. Klepeis , K. J. Schafer, T. W. Barbee, and M. Ross, Science 254, 986 (1991).

[27] O. Pfaffenzeller, D. Hohl, and P. Ballone, Phys. Rev. Lett. 74, 2599 (1995).

[28] B. Militzer, J. Low Temp. Phys. 139, 739 (2005).

[29] J. Vorberger, I. Tamblyn, B. Militzer, and S. A. Bonev, Phys. Rev. B 75, 024206 (2007).

[30] M. A. Morales, E. Schwegler, D. Ceperley, C. Pierleoni, S. Hamel, and K. Caspersen, Proc. Natl. Acad. Sci. U.S.A. 106, 1324 (2009).

[31] W. Lorenzen, B. Holst, and R. Redmer, Phys. Rev. B 84, 235109 (2011).

[32] J. M. McMahon, M. A. Morales, C. Pierleoni, and D. M. Ceperley, Rev. Mod. Phys. 84, 1607 (2012).

[33] P. Loubeyre, R. LeToullec, D. Hausermann, M. Hanfland, R. J. Hemley, H. K. Mao, and L.W. Finger, Nature (London) 383, 702 (1996).

[34] M. W. Wong, J. Am. Chem. Soc. 122, 6289 (2000).

[35] R. T. Howie, C. L. Guillaume, T. Scheler, A. F. Goncharov, and E. Gregoryanz, Phys. Rev. Lett. 108, 125501 (2012).

[36] See Supplemental Material at http://link.aps.org/ supplemental/10.1103/PhysRevLett.121.195702 for extended experimental details and figures (Figs. S1-S15), which includes Refs. [37-40].

[37] C. Prescher and V. B. Prakapenka, High Press. Res. 35, 223 (2015).

[38] V. Petříček, M. Dušek, and L. Palatinus, Z. Kristallogr. Cryst. Mater. 229, 345 (2014).

[39] M. Gauthier, Ph. Pruzan, J. C. Chervin, and J. M. Besson, Phys. Rev. B 37, 2102 (1988).

[40] S. Ninet, F. Datchi, A. M. Saitta, M. Lazzeri, and B. Canny, Phys. Rev. B 74, 104101 (2006).

[41] A. Dewaele, M. Torrent, P. Loubeyre, and M. Mezouar, Phys. Rev. B 78, 104102 (2008).

[42] Y. Akahama, and H. Kawamura, J. Phys. Conf. Ser. 215, 012195 (2010).

[43] R. T. Howie, I. B. Magdau, A. F. Goncharov, G. J. Ackland, and E. Gregoryanz, Phys. Rev. Lett. 113, 175501 (2014).

[44] S. Jiang, X. Huang, D. Duan, S. Zheng, F. Li, X. Yang, Q. Zhou, B. Liu, and T. Cui, J. Phys. Chem. C 118, 3236 (2014).

[45] R. Chellappa, D. Dattelbaum, L. Daemen, and Z. Liu, J. Phys. Conf. Ser. 500, 052008 (2014). 